03

\title{
Управление воздушным потоком вокруг цилиндрической модели с помощью вращающейся электрической дуги во внешнем магнитном поле. Часть II
}

\author{
(С) Д.С. Мунхоз, ${ }^{1}$ И.П. Завершинский, ${ }^{3}$ А.И. Климов, ${ }^{1}$ П.Н. Казанский, ${ }^{1}$ Н.Е. Молевич, ${ }^{2,3}$ \\ И.А. Моралев, ${ }^{1}$ Л.Б. Поляков, ${ }^{1}$ Д.П. Порффирьев, ${ }^{2,3}$ С.С. Сугак, ${ }^{3}$ Б.Н. Толкунов ${ }^{1}$ \\ ${ }^{1}$ Объединенный институт высоких температур РАН, \\ 124412 Москва, Россия \\ ${ }^{2}$ Физический институт им. П.Н. Лебедева РАН (филиал), \\ 443011 Самара, Россия \\ ${ }^{3}$ Самарский государственный аэрокосмический университет им. С.П. Королева, \\ 443086 Самара, Россия \\ e-mail: dasmunhoz@yahoo.com.br
}

(Поступило в Редакцию 25 октября 2016 г.)

Изучено влияние закрутки пристеночного газового потока вокруг цилиндрической модели, индуцированной магнитно-плазменным актуатором, на ее обтекание внешним дозвуковым газовым потоком.

\section{Введение}

В предыдущей работе [1] были описаны результаты экспериментальных измерений по изучению структуры и динамики пристеночного завихренного газового потока вокруг цилиндрической модели, индуцированного вращающейся электрической дугой в магнитном поле, без внешнего потока. В настоящей работе изучалось влияние закрутки пристеночного газового потока вокруг цилиндрической модели, индуцированной магнитно-плазменным актуатором (МГПА), на ее обтекание внешним дозвуковым газовым потоком $(M<0.2$, $\left.\operatorname{Re}<9 \cdot 10^{4}\right)$. В эксперименте измерялись: профиль потока вокруг цилиндра, аэродинамические силы, действующие на цилиндр (сила сопротивления и подъемная сила). Для измерения профиля скорости возмущенного потока вокруг цилиндра использовались высокоскоростная съемка, теневой и PIV-методы.

Для изучения обтекания цилиндра с работающим МГПА использовалась модель с четырьмя разрядными промежутками (рис. 1). В этой модели плазма максимально покрывала ее поверхность, что позволило измерить аэродинамические силы, действующие на эту модель.

\section{Режим вращения дугового разряда во внешнем магнитном поле на цилиндрической модели, обтекаемой воздушным потоком}

На рис. 2 показан интегральный снимок обтекания цилиндрической модели с вращающейся дугой и четырьмя разрядными промежутками. Обостритель разряда на модели располагается в положении угла атаки $\alpha=5^{\circ}$ по отношению к направлению воздушного потока. Величина максимального импульсного тока на поверхно- сти модели достигает значения порядка $I_{\max } \sim 16 \mathrm{~A} \mathrm{в}$ этом эксперименте. Длительность силового импульса составляет $T_{i}<3 \mathrm{~ms}$. Характерное число Рейнольдса набегающего потока $\operatorname{Re} \approx 6 \cdot 10^{3}\left(V_{\infty} \approx 5 \mathrm{~m} / \mathrm{s}\right)$.

Экспериментальные данные по измерению линейной скорости вращающейся дуги по поверхности обтекаемого цилиндра, полученные в этом эксперименте, приведены на рис. 3. Из рисунка следует, что максимальная достигнутая скорость дуги составляет $V_{i} \approx 20 \mathrm{~m} / \mathrm{s}$ при величине максимального импульсного тока порядка $16 \mathrm{~A}$. Обнаружено, что значение скорости вращения дуги без внешнего потока и с ним близки друг другу.

\section{PIV-измерения поля течения вокруг обтекаемой модели с работающим МГПА}

Усредненное поле скоростей вокруг цилиндрической модели с работающим МГПА обтекаемым внешним потоком со скоростью $V_{\infty} \approx 6 \mathrm{~m} / \mathrm{s}\left(\operatorname{Re} \approx 7.4 \cdot 10^{3}\right)$, показано на рис. 4. В этих экспериментах использовался источник питания с постоянным током $I_{d}=0.64 \mathrm{~A}$. Из рисунка следует, что в этом эксперименте реализуется значительное изменение положения точки отрыва потока на модели. Смещение точки отрыва потока может достигать угла $\Delta \theta=40^{\circ}$.

Для того чтобы изучить влияние МГПА на отрыв потока, на модели использовался режим внешней синхронизации видеосьемки и PIV-установки. Измеренная частота вращения дугового разряда в этом эксперименте составляла $F_{r} \approx 290 \mathrm{~Hz}$. В этом эксперименте использовался импульсно-периодический разряд со следующими характеристиками: $F_{i}=7 \mathrm{~Hz}, U_{\max }<15 \mathrm{kV}, I_{\max }<30 \mathrm{~A}$, $P_{\max }<70 \mathrm{~kW}, P_{\operatorname{med}}=200 \mathrm{~W}$. На рис. 5 показаны типичные сигналы напряжения и тока, измеренные на разрядном промежутке МГПА. Скорость воздушного 

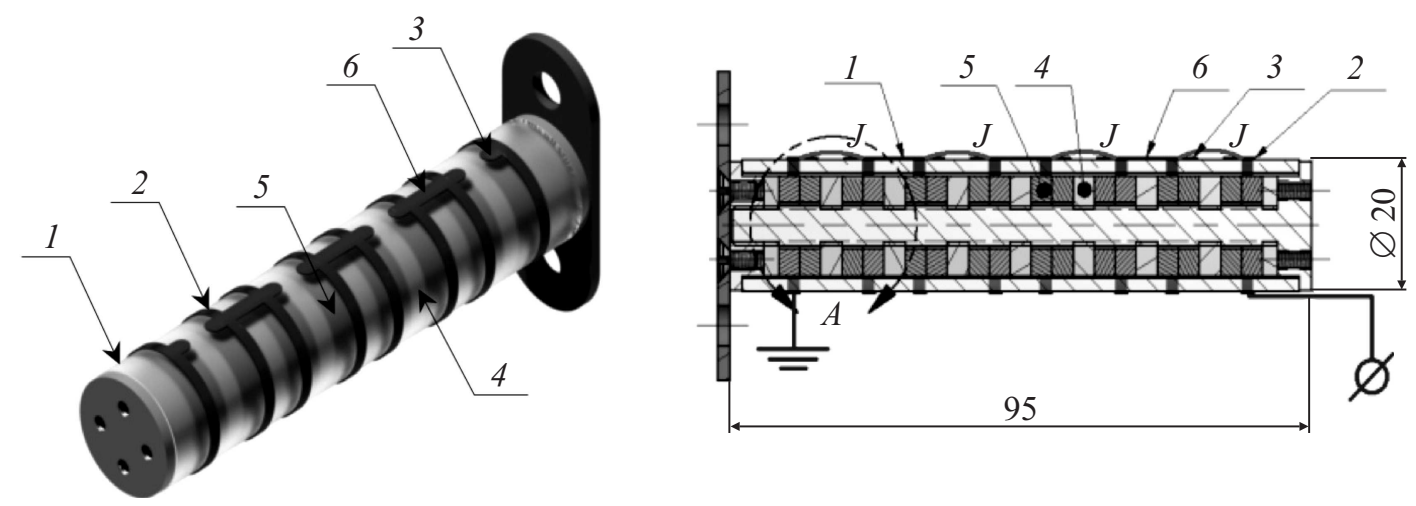

Рис. 1. Общий вид и схема цилиндрической модели с многозвенным МГД актуатором: 1 - кварцевая трубка, $2-$ медное кольцо, 3 - обостритель, 4 - диэлектрическая гайка и шпилька из полиамида, $5-\mathrm{Nd}-\mathrm{Fe}-\mathrm{B}$-магниты, $6-$ соединяющая перемычка между электродными кольцами.
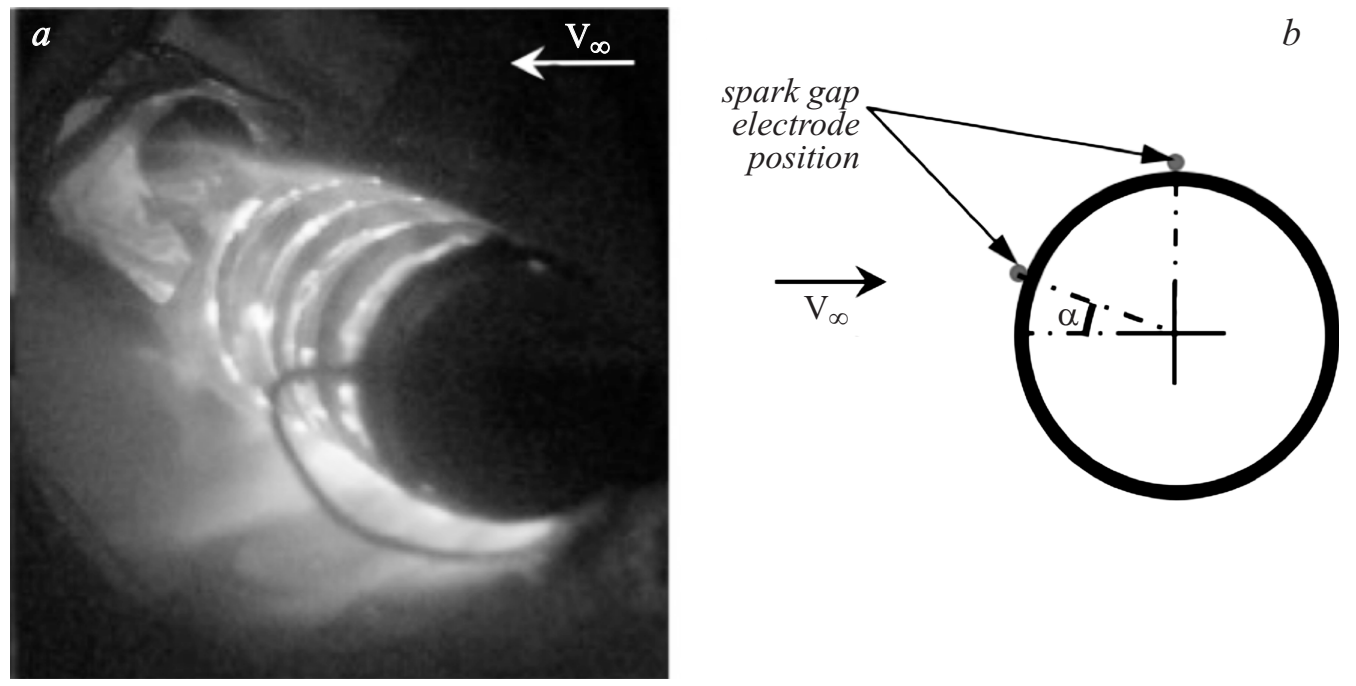

Рис. 2. Интегральный кадр обтекания модели с вращающейся дугой с четырьмя разрядными промежутками (a). Скорость воздушного потока порядка $\operatorname{Re} \sim 6.2 \cdot 10^{3}\left(V_{\infty} \sim 5 \mathrm{~m} / \mathrm{s}\right)$. Схема расположения обострителей на цилиндрической модели в позиции $\alpha=5^{\circ}$ или $\alpha=90^{\circ}$ по отношению к направлению воздушного потока $(b)$.

потока составляла $\operatorname{Re}=8 \cdot 10^{3}\left(V_{\infty}=6.5 \mathrm{~m} / \mathrm{s}\right)$. Обостритель находился в положении $\alpha=5^{\circ}$. Поле скоростей вблизи обтекаемой цилиндрической модели с работающим МГПА во внешнем потоке в этом режиме показано на рис. 6. Количество измерений для усреднения каждой PIV-картины - 70. При этих условиях обнаружено значительное влияние вращающейся дуги на обтекание самой модели. На рис. 6 показано поле течения вокруг обтекаемой модели с МГПА при завершении второго оборота дуги вокруг модели. В этом режиме возмущается все поле скоростей вокруг модели, в том числе в ее головной части и донной частях. На рисунке имеется также синхронное видеоизображение вращающегося разряда в соответствующий момент времени. Из анализа этого рисунка также следует, что изменение циркуляции потока вокруг модели происходит как в головной части дугового разряда $(1)$, так и в его хво- стовой части (2). Отметим, что зона стимулированной циркуляции пристеночного потока имеется и в донной области модели. При этом практически отсутствует отрыв потока в этой области вплоть до углов $\theta \approx 260^{\circ}$. Этот результат позволяет определить усредненное поле индуцированной завихренности вокруг модели в данном эксперименте. Анализ теневых снимков, температуры дуги [2-5] и PIV-кадров позволяет сделать вывод, что во всем слое пристеночного потока вокруг цилиндра, за исключением зоны вблизи электрической дуги, реализуется режим слабо сжимаемого течения (имеются малые изменения плотности, $\delta \rho / \rho_{0} \ll 1$, где $\rho_{0}-$ плотность воздуха, и мало изменение скорости индукционного потока, $\delta V / V_{0} \ll 1$, где $V_{0}-$ скорость потока).

Оценим стимулированную циркуляцию потока вблизи цилиндра с работающим МГПА в этом эксперименте (рис. 6). Величина стимулированной циркуляции Г 


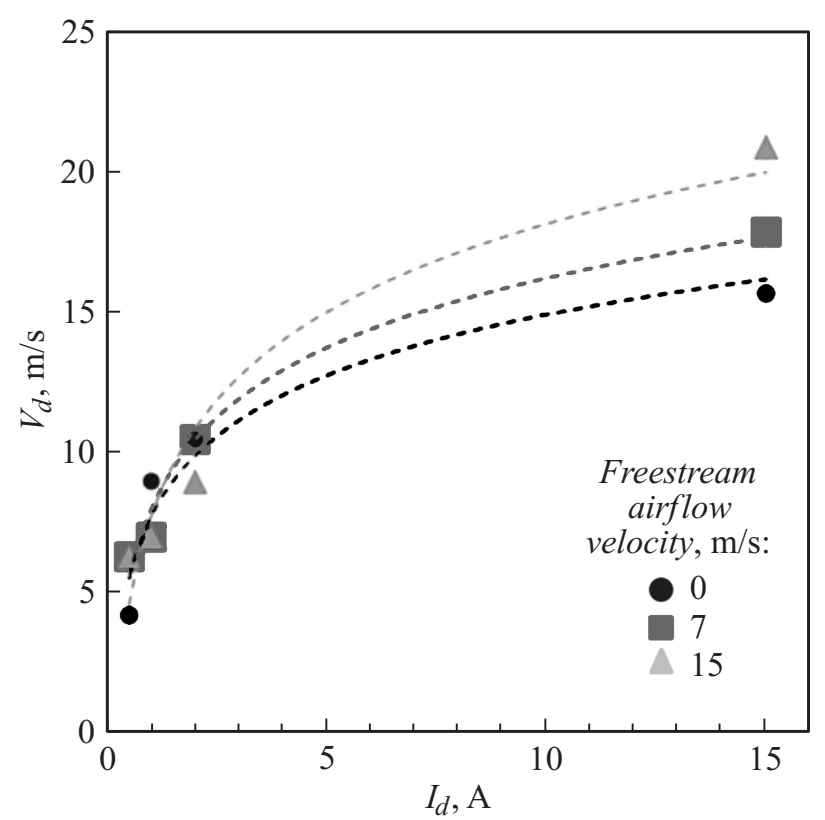

Рис. 3. Зависимость линейной скорости вращающейся дуги $V_{d}$ на поверхности от величины разрядного тока $I_{d}$.

вблизи поверхности цилиндра определялась с помощью следующего выражения [1]:

$$
\begin{aligned}
\Gamma & =\iint_{S}(\nabla \times V) d S \\
& =\int_{0}^{2 \pi} \int_{R_{0}}^{\left(R_{0}+h\right)}(\nabla \times V) R_{0} d \theta d r \approx 0.095 \mathrm{~m}^{2} / \mathrm{s},
\end{aligned}
$$

где $R_{0}=10 \mathrm{~mm}$ и $h=4 \mathrm{~mm}$.

Значение стимулированной завихренности потока вокруг модели $([1],(2))$ получилось равной $\xi \approx 2000 \mathrm{~s}^{-1}$, где

$$
\xi=\nabla \times V=\operatorname{Rot} V=2 \omega .
$$

Такое большое значение завихренности $\xi$ по сравнению с работой [1] было получено вследствие использования разряда с высокой величиной импульсного тока в эксперименте.

\section{Измерение силовых характеристик модели}

Измерения аэродинамических характеристик цилиндрической модели с работающим МГПА проводились на аэродинамической трубе АДТ-2 [1]. На этой модели размещались четыре разрядных промежутка. Параметры настоящих экспериментов были следующие: импульсный ток $I_{\max } \approx 30 \mathrm{~A}$, длительность импульса $T_{i}<1 \mathrm{~ms}$, частота повторения импульсов $F_{i}<1200 \mathrm{~Hz}$, напряжение разряда $U_{d}<4.4 . \mathrm{kV}$, скорость воздушного потока изменялась от 10 до $74 \mathrm{~m} / \mathrm{s}$, инициатор разряда располагался в положениях $\alpha=5^{\circ}$ или $90^{\circ}$.

Было обнаружено, что:

1. Подъемная сила была практически равной нулю, когда МГПА не был включен.

2. Сила сопротивления не изменялась при включении МГПА (с точностью до 10\%).

3. Подъемная сила достигала бо́льших значений $L=0.37 \mathrm{~N}$ при включении МГПА.

Типичные сигналы с датчика подъемной силы показаны на рис. 7,a. Обнаружено, что величина подъемной силы $L$ для модели с работающим МГПА всегда отлична от нуля. Ее величина зависит от величины стимулированной циркуляции пристеночного потока на модели.

При положении инициатора на цилиндре $\alpha=90^{\circ}$ величина подъемной силы увеличивалась до $L=0.45 \mathrm{~N}$.

Оценим величину безразмерного коэффициента подъемной силы

$$
C_{L, \exp }=L /\left(\rho_{\infty} V_{\infty}^{2} w d / 2\right) \approx 0.3,
$$

где размер плазменной зоны между четырьмя электродными промежутками на цилиндре $w=40 \mathrm{~mm}$, диаметр модели $d=20 \mathrm{~mm}$, величина измеренной подъемной силы $L=0.37 \mathrm{~N}$ и $V_{\infty}=52 \mathrm{~m} / \mathrm{s}\left(\operatorname{Re} \approx 6.4 \cdot 10^{4}\right)$.

При положении инициатора на цилиндре $\alpha=90^{\circ}$ оценка величины $C_{L}$ достигает величины 0.6 .

\section{Обсуждение полученных результатов}

На рис. 6 отчетливо видно, что имеется несимметричное обтекание цилиндра, вызванное индуцированной циркуляцией потока вокруг модели, созданного вращающейся электрической дугой. Такое несимметричное обтекание модели внешним газовым потоком может привести к возникновению боковой подъемной силы цилиндра. Практическое отсутствие отрыва потока на модели и слабо сжимаемый режим течения пристеночного газа в этом эксперименте [1] позволяют сделать упрощенные оценки величины подъемной силы (модифицированной силы Жуковского) и величины коэффициента подъемной силы $C_{l, t}$ за счет создания стимулированной циркуляции Г на основании формулы из работы [6]:

$$
C_{L, t}(t)=2 \Gamma(t) /\left(V_{\infty} d\right)+\{2 R[d \Gamma(t) / d t]\} /\left(V_{\infty}^{2}\right)+C_{l w}^{(v)},
$$

где первый член - квазистационарный коэффициент подъемной силы $C_{L, u}$, второй - нестационарная сила и третий - вихревая сила. Будем считать, что $C_{L, u}$ можно рассматривать как первое приближение для определения полного коэффициента подъема силы цилиндрической модели. Его величина достигает значения

$$
C_{L, u}=2 \Gamma /\left(V_{\infty} d\right) \approx 0.2,
$$

где $\Gamma=0.095 \mathrm{~m}^{2} / \mathrm{s}(3), V_{\infty}=42 \mathrm{~m} / \mathrm{s}\left(\operatorname{Re} \approx 5.2 \cdot 10^{4}\right)$ 


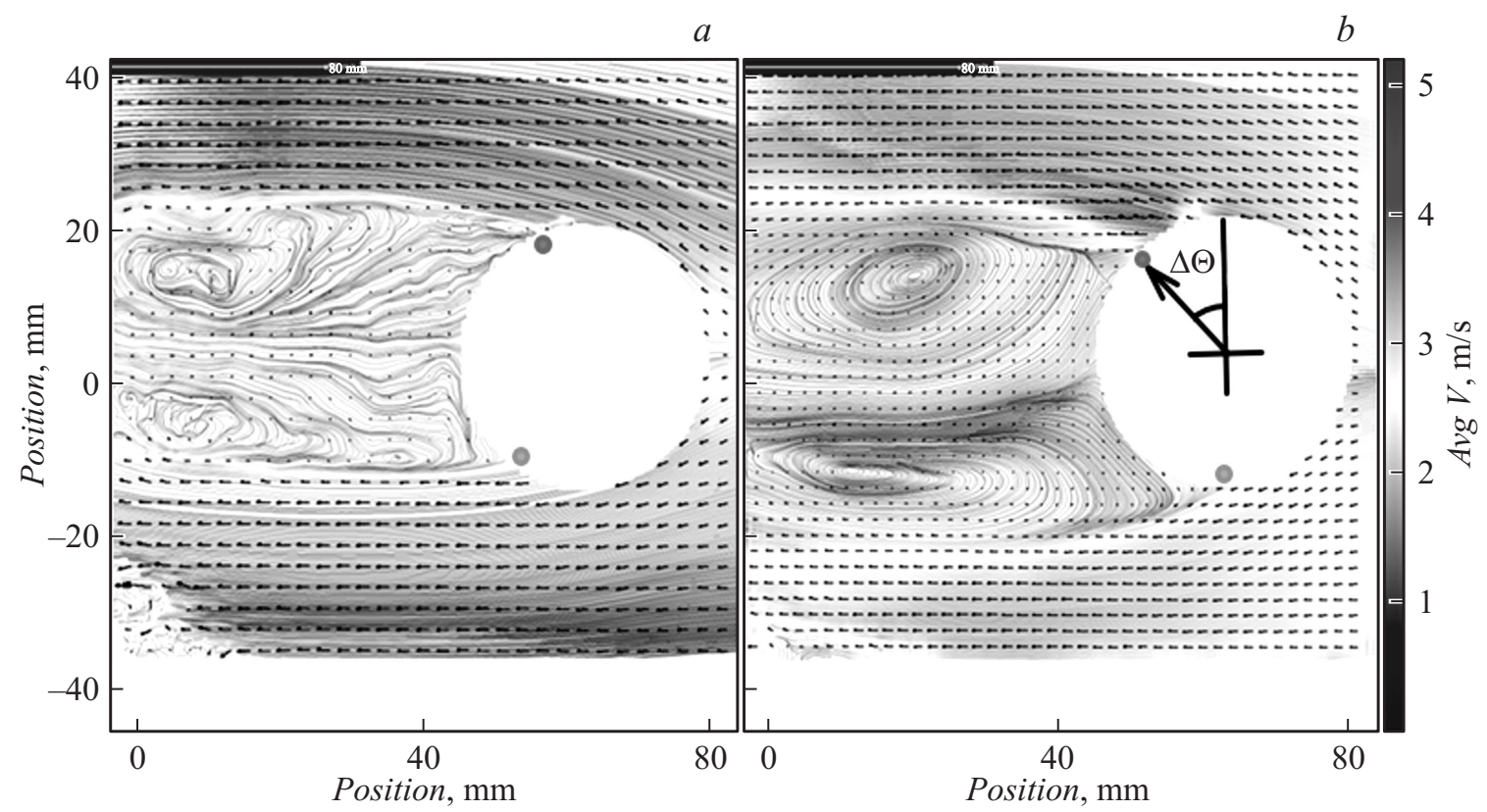

Рис. 4. Среднее поле скоростей вокруг цилиндрической модели, измеренное PIV-методом. $a-\operatorname{Re} \approx 7.4 \cdot 10^{3}\left(V_{\infty} \approx 6 \mathrm{~m} / \mathrm{s}\right) ; b-$ режим с работающим МГПА, разряд постоянного тока $I_{d} \approx 0.64 \mathrm{~A}$.

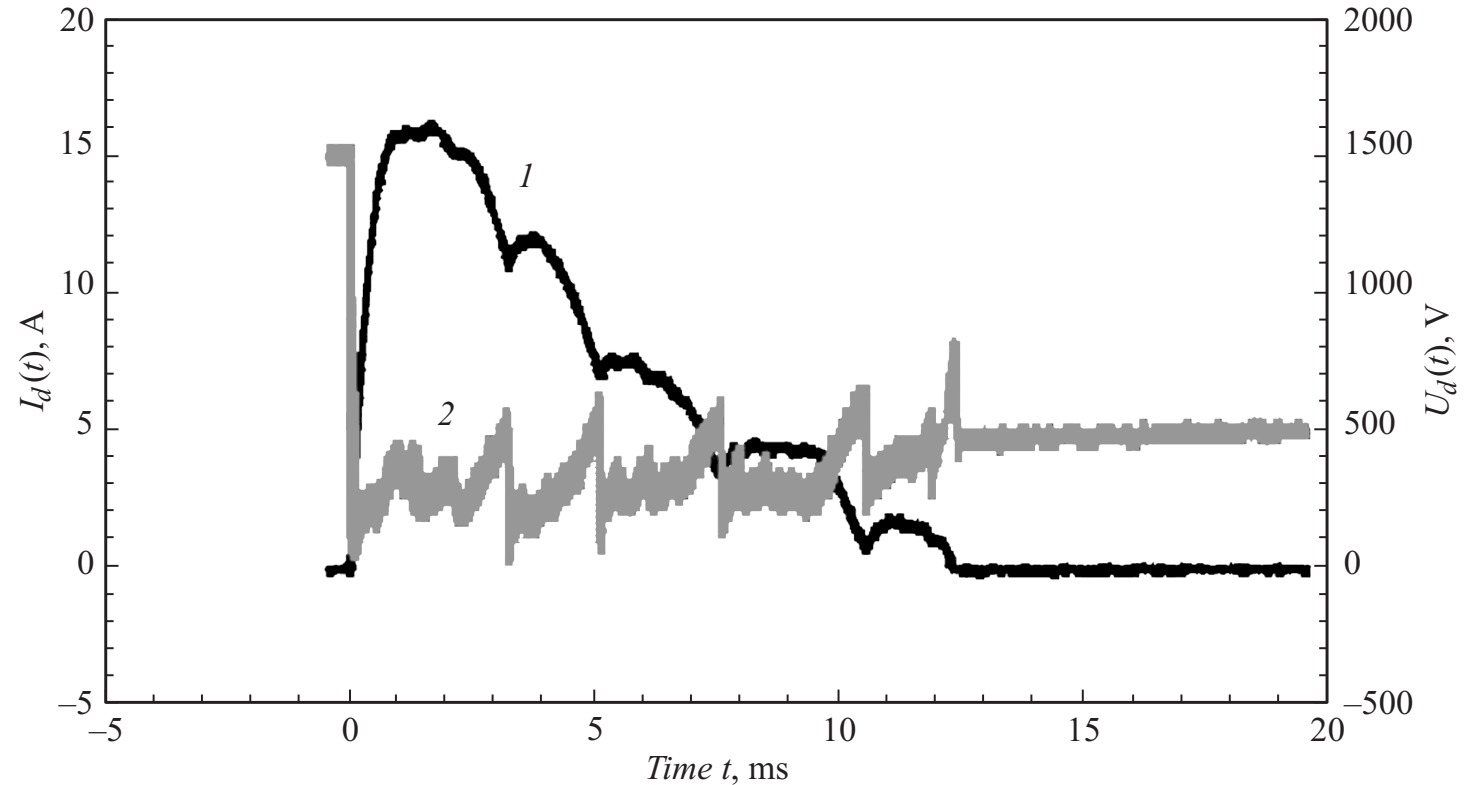

Рис. 5. Импульсный ток $I_{d}(1)$ и напряжение $U_{d}(2)$ в МГПА.

Удивительно, что величина расчетного значения $C_{L, u}$ вычисленная по формуле (5) оказалась близкой к экспериментальному значению $C_{L, \exp } \approx 0.3$. Их близкие значения позволяют предположить, что наши допущения, используемые при оценке $C_{L, u}$, являются правдоподобными. Детальное изучение этого вопроса будет проведено в наших следующих работах.

Можно оценить величину безразмерного коэффициента числа Струхаля в этом эксперименте

$$
\mathrm{St}^{*}=f D / V=0.4
$$

при размере плазменной зоны между четырьмя электродными промежутками на цилиндре $w=40 \mathrm{~mm}$, диаметре модели $D=20 \mathrm{~mm}$, положении инициатора разряда $\alpha=5^{\circ}$, величине подъемной силы $L=0.37 \mathrm{~N}$, частоте повторения разрядных импульсов $F_{i} \approx 1 \mathrm{kHz}$ и $\operatorname{Re} \approx 6.4 \cdot 10^{4} .\left(V_{\infty}=52 \mathrm{~m} / \mathrm{s}\right)$.

При положении инициатора $\alpha=90^{\circ}$ и величине $C_{L} \approx 0.6$ число Струхаля достигает $\mathrm{St}^{*}=0.25$.

Отсюда следует, что экспериментальные значения чисел Струхаля $\mathrm{St}^{*}=0.25-0.4$ близки к нижнему кри- 

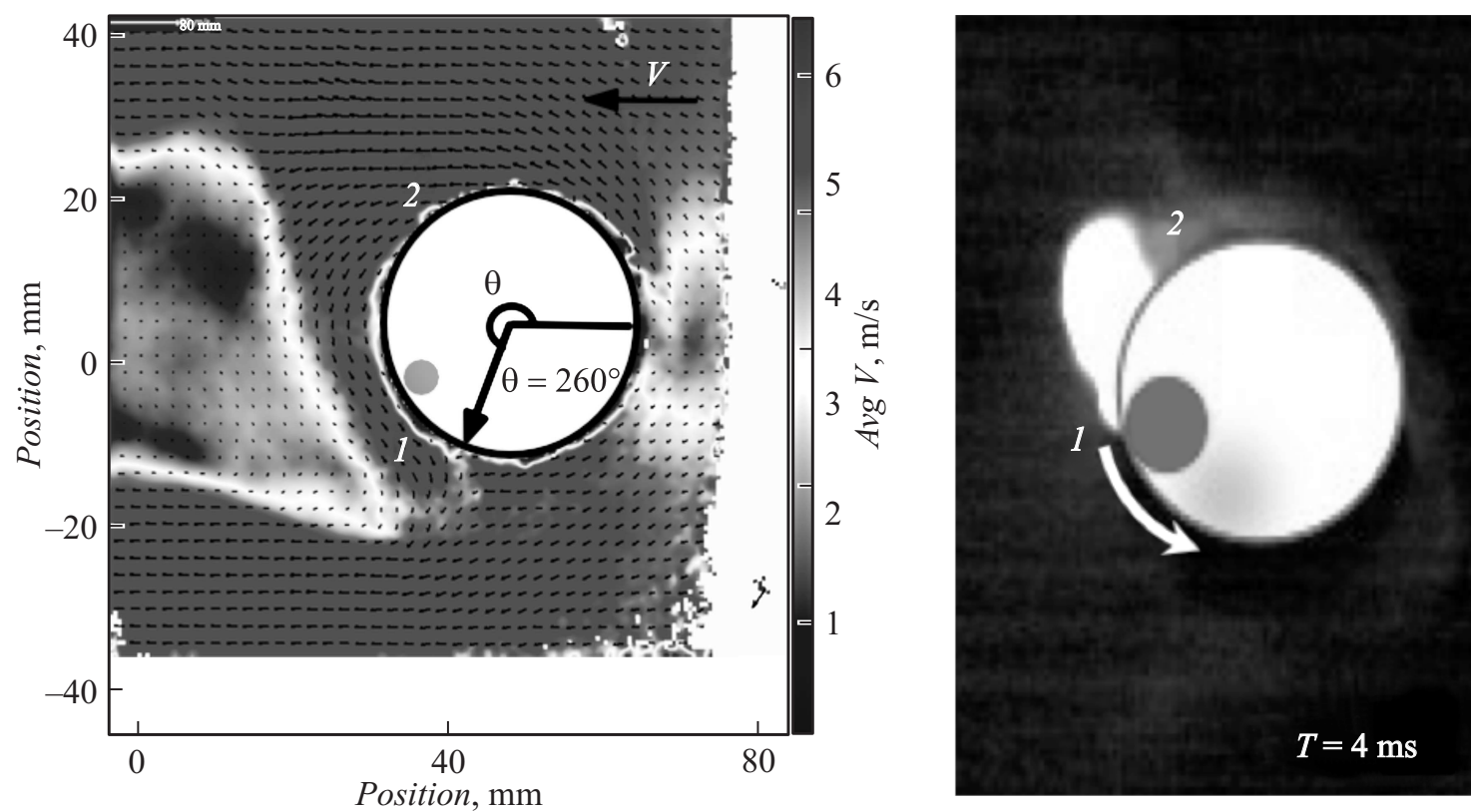

Рис. 6. Усредненная PIV-картина поля скоростей вокруг цилиндра с вращающейся плазменной дугой. Характерное число Рейнольдса набегающего потока $\operatorname{Re}=8 \cdot 10^{3}\left(V_{\infty} \approx 6.5 \mathrm{~m} / \mathrm{s}\right)$. Время задержки после включения разряда $T_{2}=4 \mathrm{~ms}$.
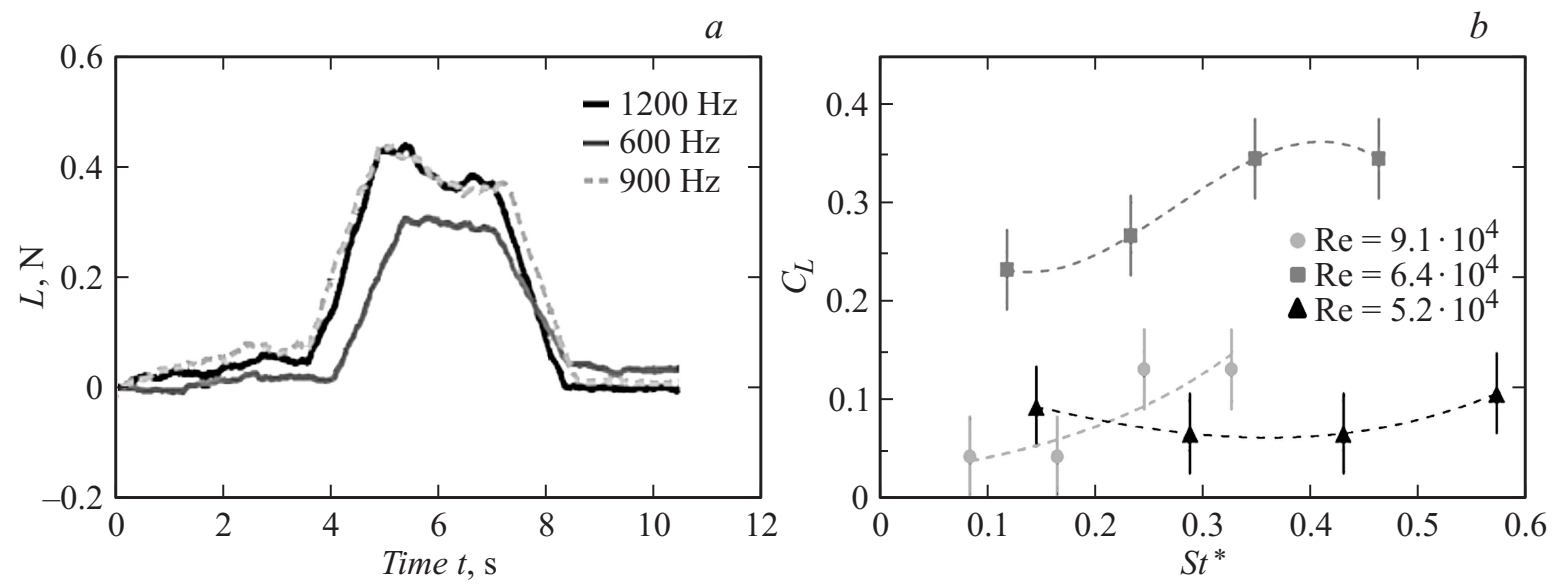

Рис. 7. Подъемная сила $L$ на цилиндрической модели при включении МПА $(t<3.5 \mathrm{~s}$ и $t>9 \mathrm{~s})$ и его выключении $(5.5 \mathrm{~s}<t<7 \mathrm{~s})$ и $V_{\infty}=42 \mathrm{~m} / \mathrm{s}(a)$. Зависимость коэффициента подъемной силы $C_{L}$ от числа Струхаля $\mathrm{St}^{*}$ импульсно-периодического разряда $(b)$.

тическому его значению $\mathrm{St}^{*}=0.3-0.5$, при котором происходит отрыв потока на цилиндре и формирование крупномасштабных вихревых возмущений [7]. Поэтому в наших экспериментах с работающим МГПА наблюдается формирование несимметричных крупномасштабных вихрей за обтекаемым цилиндром, которые могут приводить к несимметричному обтеканию самой модели (рис. 4,6).

\section{Сравнение полученных результатов с численным расчетом}

В работе [4] были проведены теоретические расчеты обтекания цилиндра с цилиндрической зоной энерго- выделения малого диаметра, вращающейся вдоль ее поверхности, при условиях близких к настоящей работе, а именно числах Маха набегающего потока $M=0.15$ и 0.21 и погонной мощности $100 \mathrm{~W} / \mathrm{cm}$. При этом движущаяся электрическая дуга во внешнем магнитном поле моделировалась этой зоной теплового энерговыделения. Из расчетов следует, что область энерговыделения приводит к нарушению симметрии обтекания цилиндрической модели, появлению стимулированной циркуляции вокруг модели и подъемной силы. Там же было получено значение усредненной по времени стимулированной циркуляции потока вблизи модели.

Анализ наших экспериментальных результатов и теоретических результатов [8] показывает, что они качественно близки друг другу, но имеются количественные 
различия между ними. Эти различия могут быть обусловлены разными причинами, в том числе трехмерным характером структуры электрической дуги во внешнем потоке, упрощенным моделированием канала электрической дуги и его движением во внешнем магнитном поле.

В заключении можно отметить, что созданный МГПА способен создавать вихревой пристеночный поток вокруг цилиндрической модели, который влияет на ее обтекание внешним дозвуковым потоком $(M<0.2$, $\left.\operatorname{Re}<9 \cdot 10^{4}\right)$. Это утверждение подтверждается результатами прямых измерений с помощью PIV и теневого методов, весовыми измерениями, а также результатами теоретического расчета [4]. Обнаружено что появление подъемной силы цилиндра происходит за счет создания стимулированной циркуляции потока вокруг него [4]. Величина подъемной силы в эксперименте достигала максимальных значений порядка $L=0.37-0.45 \mathrm{~N}$. При этом величина коэффициента подъемной силы $C_{L}$ достигала $0.3-0.6$.

Работа поддержана Международным фондом CNPq, Conselho Nacional de Desenvolvimento Cienífico e Tecnológico (Бразилия), в рамках Программы „Наука без границ“, 2012-2015 гг., грант CNPq 200205/2012-9.

\section{Список литературы}

[1] Мунхоз Д.С., Битюрин В.А., Климов А.И., Казанский П.Н., Моралев И.А., Поляков Л.Б., Толкунов Б.Н. // ЖТФ. 2017. Т. 83. Вып. 7.

[2] Автореф. канд. дис. Моралев И.А. // Взаимодействие газоразрядной плазмы с закрученными течениями, кандидатская диссертация. ОИВТ РАН. 2010.

[3] Автореф. канд. дис. Бочаров А.Н. // Физические и численные модели магнитоплазменной аэродинамики. ОИВТ PAH, 2011.

[4] Битюрин В.А., Завершинский И.П., Климов А.И., Молевич Н.Е., Моралев И.А., Мунхоз Д.С., Поляков Л.Б., Порфирьев Д.П., Судак С.С. // ТВТ. 2016. Т. 54. № 4. С. 1-4.

[5] Bityurin V., Bocharov A. // Proc. of the $39^{\text {th }}$ Aerospace Sciences Meeting and Exhibit Reno. USA, AIAA-2001-0793.

[6] Yuan J.K., Olinger D.J. // Circulation methods for unsteady aerodynamics flows, $20^{\text {th }}$ AIAA Applied Aerodynamics Conf. Sessions of Unsteady Aerodynamics. St. Louis, 2002. AIAA2002-3057.

[7] Автореф. канд. дис. Казанский П.Н. // Управление потоком вблизи аэродинамических тел с помощью плазменного высокочастотного актуатора. МЭИ 2012.

[8] Leroy A., Podlinski J., Devinant P., Aubrun S. // Proc of the Abstract $6^{\text {th }}$ European Conf. for Aeronautics and Space Sciences (EUCASS). Krakow. 2015. 